\title{
General Framework of Compressive Sampling and its Applications for Signal and Image Compression
}

\author{
A Random Approach \\ Prabhat Thakur \\ Jaypee University of Information Technology, Waknaghat, Solan (H.P.)
}

\begin{abstract}
Compressive sampling emerged as a very useful random protocol and has become an active research area for almost a decade. Compressive sampling allows us to sample a signal below Shannon Nyquist rate and assures its successful reconstruction if the signal is sparse. In this paper we used compressive sampling for arbitrary signal and image compression and successfully reconstructed them by solving 11 norm optimization problem. We also showed that compressive sampling can be implemented if signal is sparse and incoherent through simulations.
\end{abstract}

Keywords: Basis Function, Compressive Sampling, Incoherent Signal, l1-norm, Sparse Signal.

\section{INTRODUCTION}

$\mathrm{T}$

Today we are moving towards digital domains, but origination of a signal is in analog domain most of the times. So analog to digital conversion systems are required but these systems follow some criteria i.e. sampling frequency should be greater than twice of the analog signal frequency (Shannon Nyquist Criteria) [1]. But if the frequency of signal will be very high then it will be very inconvenient to use Nyquist criteria because number of samples will be very large. Storage and processing of such large number of samples will be costly or sometimes infeasible.

But if somehow we can overcome a Shannon Nyquist criterion that is if we can reconstruct original signal by using very less number of samples as compared to the Nyquist criteria, then problem of storage and processing of large data can be solved. This problem may solved by compressive sampling [2-4] a random approach if signal is sparse in some domain.

Compressive Sampling uses very less number of samples as compared to Shannon Nyquist rate which reduces the hardware and software loads and then signal is recovered by using various recovery mechanisms [5-7]. Compressive sampling uses a random matrix to form out linear random projections of signals with most of the desired information. It is possible due to two properties of signal i.e. sparsity and incoherence [8]. Sparsity refers to the property of signal according to which information present in signal is very less as compare to the bandwidth occupied by the signal. Incoherence is property
Journal of Technology Management for Growing Economies Vol. 6, No. 1 April 2015 pp. 7-14

\section{CHITKARA 音 UNIVERSITY}

(C) 2015 by Chitkara University. All Rights Reserved. 

domain in which signal is sparse. If signal will be more sparse i.e. low sparsity level, its reconstruction will be better as compared to less sparse signal.

The paper is organized as follows. Section II provides some background on Compressive Sampling, Mathematical Model and Signal Reconstruction by solving Optimization problems. In Section III we use compressive sampling for signal and image compression and its successful reconstruction. Section IV presents simulation results on its performance.

\section{BACKGROUND}

\section{Compressive Sampling}

Emerging theory of compressive sampling (CS) allows us to project random measurements of signal of interest so that we can sample the signal at information rate rather than its ambient data rate. This reduces the number of samples to represent a signal. Reduced number of samples can be stored easily and processing of such small number of samples can be performed efficiently. But to apply compressive sampling on the signal, signal should be sparse and incoherent.

Sparse Signal: A signal is said to be sparse if only some of the components have significant magnitude and all other components have insignificant magnitude i.e. closer to zero.

Incoherent Signal: Sparse signal that can be transformed into desired domain are known as incoherent signal.

\section{Mathematical Approach for Compression}

Consider a signal which is sparse. is said to be sparse if it can be represented as a linear combination of basis functions where some of the coefficient's magnitude is significant and all others have zero magnitude

$$
\begin{aligned}
& r=\psi c \\
& \psi-\text { Basis Functions } \\
& c \text { - Basis Coefficients }
\end{aligned}
$$

For compressive sampling a random matrix $\varnothing$ need to project random projections or random measurements

$$
b=\varnothing r
$$

$b$ - Random Measurement vector

\section{Reconstruction}

Now we need to reconstruct back $r$ form $b$

$$
b=\varnothing \psi c
$$


By solving above equation we can find basis coefficient $c$.

General

Information of leads us towards recovery solution

$$
r=\psi c
$$

\section{Optimization Problem Formulation}

The equation we have to solve i.e. (1) is an underdetermined system as number of equations is less than number of unknowns. So we need to use norm minimization techniques to solve above problem.

Mathematically norm provides total size or positive lengths of all vectors in a vector space or matrices. Generally Norm $\mathrm{n}$ of vector $\mathrm{x}$ is defined as:

$$
\|\mathrm{x}\|_{\mathrm{n}}=\sqrt{\sum_{\mathrm{i}}}\|\mathrm{x}\|^{\mathrm{n}}
$$

Where, $n \in R$

Frequently using norms are 10,11, 12 but here we use 11 norm.

11norm : 11 norm is defined as : $\|x\|_{1}=\sum_{\mathrm{i}}|\mathrm{x}|$

11 optimization problem is formulated as:

$$
\min || \mathrm{x} \|_{1} \text { subject to }|\mathrm{Ax}=\mathrm{b}|
$$

Above problem can be solved using least square optimization

$$
\mathrm{x}=\mathrm{A}^{+} \mathrm{b} \text {, }
$$

Where $\boldsymbol{A}^{+}$-Psuedoinverse of $\mathrm{A}$

Even though this method is easy to compute but it is not necessary that it provides best solution. That is why we use 11 norm optimization.

So our optimization problem is formulated as:

$$
\min || \boldsymbol{c} \|_{1} \text { subject to }|(\emptyset \psi) \boldsymbol{c}=\boldsymbol{b}|
$$

\section{1 optimization solution}

11 optimization problems can be solved by using these two

types of algorithms

1. Greedy Type- Orthogonal Matching Pursuit, Basis Pursuit.

Orthogonal matching pursuit is a greedy-type algorithm as it selects the one index regarded as the optimal decision at each iteration.

Basis pursuit is a technique where signal is decomposed into an optimal superposition of dictionary elements. Optimization criteria is 11 norm of coefficients

Journal of Technology Management for Growing Economies, Volume 6, Number 1, April 2015 
Thakur, $\mathrm{P}$.

2. Gradient Type-Primal Dual Interior Point.

In this algorithm we perform some changes into 11 norm and it is mainly used when innovation components are more.

\section{APPLICATIONS IN SIGNAL AND IMAGE}

Reduced load on hardware and software leads us to use compressive sampling in all possible fields.e.g. Signal compression, Image compression, Speech compression, audio and video compression, wireless sensor networks etc. But here we apply compressive sampling on one dimensional signal and 2 dimensional Image signal.

\section{Signal Compression and Recovery}

Number of times we deal with one dimensional signals such as audio signals speech signals and we need to sample these signal for performing some digital operation on these signal. Less number of samples can be processed easily with reduced processing time. So we go for compressive sampling of such signals if they are sparse. Complete recovery of signal relies on sparsity level (SL) and compression ratio (CR). Sparsity level is number of components having significant magnitude. Compression ratio is the ratio that up to what level we have compressed the signal e.g. N/10 where is total number of samples present in the signal.

If sparsity level is low, recovery will be better. If compression ratio is more, recovery will be better.

Consider a one dimensional signal $r$ having length $\mathrm{n}$

$$
r_{n^{* 1}}
$$

$r_{\mathrm{n}^{*} 1}$ can be represented with the help of basis functions and

its coefficients

$$
\left.\mathrm{r}_{\mathrm{n}^{* 1}}\right)=\psi_{\mathrm{n}^{*} \mathrm{n}} \mathrm{c}_{\mathrm{n}^{* 1}}
$$

$\psi_{n^{*} n}-n^{*} n$ Matrix of basis function

$\mathrm{c}_{\mathrm{n}^{* 1}}-\mathrm{n} * 1$ Vector of basis coefficients

For random measurements after random sampling we use measurement matrix $\varnothing_{m^{*} n}$

$$
\mathrm{b}_{n^{*} 1}=\varnothing_{m^{*} n} \mathrm{r}_{n * 1}
$$

$$
\varnothing_{\mathrm{m}^{* \mathrm{n}}}-\text { Measurement Matrix }
$$

Journal of Technology Management for Growing Economies, Volume 6, Number 1, April 2015 


$$
b_{n^{*} 1}=\left(\varnothing_{m^{*} n} \psi_{n^{*} n}\right) c_{n^{*} 1}
$$

Above equation needs to solved using 11 norm optimization.

11 norm optimization problem is formulated as :

$$
\min \left\|c_{n^{*} I}\right\| \text { subject to }\left(\varnothing_{m^{*} n} \Psi_{n^{*} n}\right) \mathrm{c}_{n^{*} 1}=\mathrm{b}_{n^{*} 1}
$$

Reconstruction using above solution

\section{Recovery Error (rerr)}

Recovery error gives us the error for successful recovery and defined as:

$$
\operatorname{Rerr}=\frac{1}{\text { length }(r)} \sum_{i}^{\text {length }(r)}(\hat{r}-r)
$$

\section{Image Compression and Recovery}

Image can also be compressed using its Fourier or wavelet domain where image shows some sparse nature. Mathematical approach for image remains same as for signals but we choose basis functions either on Fourier or Wavelet domain .Instead of a one dimensional vector we deal with a two dimensional matrix.

\section{SIMULATIONS AND RESULTS}

For implementation of all algorithms, we used Matrix Laboratory on a standard computer. 11-magic toolbox is used to achieve the solution of 11-norm optimization problems.

\section{Signal Reconstruction}

We considered a signal in time domain and make it sparse in frequency domain by taking all frequency domain coefficients zero which are below some threshold value. Here threshold value is assumed as one fifth of the maximum amplitude of the coefficients. We used same procedure used for signal compression and recovery of original signal in section III-A. We sampled the signal using sampling rate which is ten times less than Nyquist rate and successfully reconstructed the original signal as shown in Fig.1. Here we varied the sparsity level of signal and analyze its results on recovery error. In addition to this we also analyzed the effect of variation of compression ratio on recovery error. 
Thakur, P.

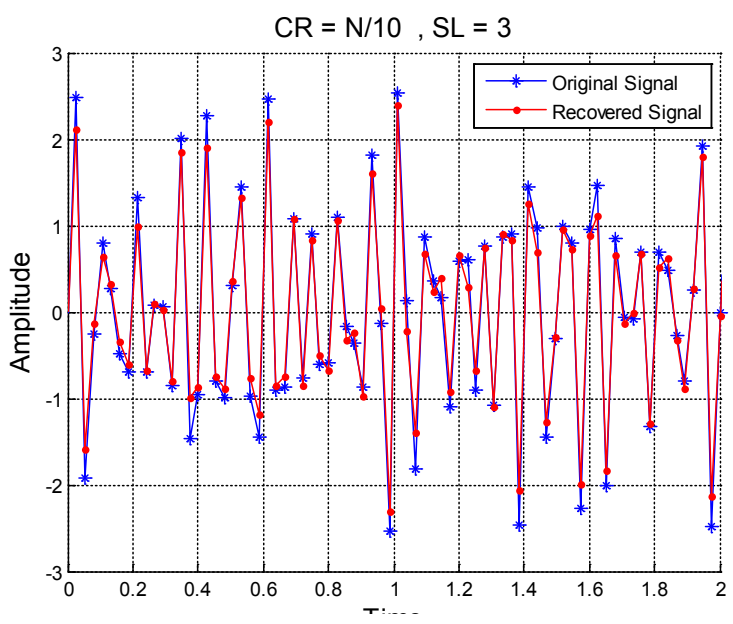

Figure 1: Original signal versus Recovered Signal

Fig.2. explains the behavior of recovery error with variation in compression ratio for different sparsity levels. It is clear that with increase in compression ratio, recovery error increases. More the sparsity level is, more the recovery error is. So it conforms that the compressive sampling can be implemented on the signals having sparse nature otherwise recovery or reconstruction cannot be done successfully. Recovery error relies on sparsity level and compression ratio. If signal is sparser, can be compressed more and can be reconstructed successfully.

\section{Image Reconstruction}

Further we compress the image by taking random measurements and recover the image using least square method (LSM) and basis pursuit (BP, an algorithm to solve 11 norm optimization problem)

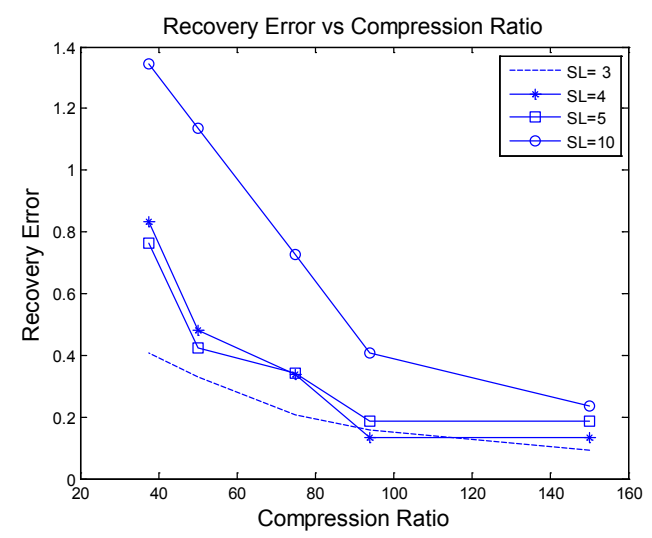

Figure 2: Recovery error versus compression ratio with different sparsity level

Journal of Technology Management for Growing Economies, Volume 6, Number 1, April 2015 


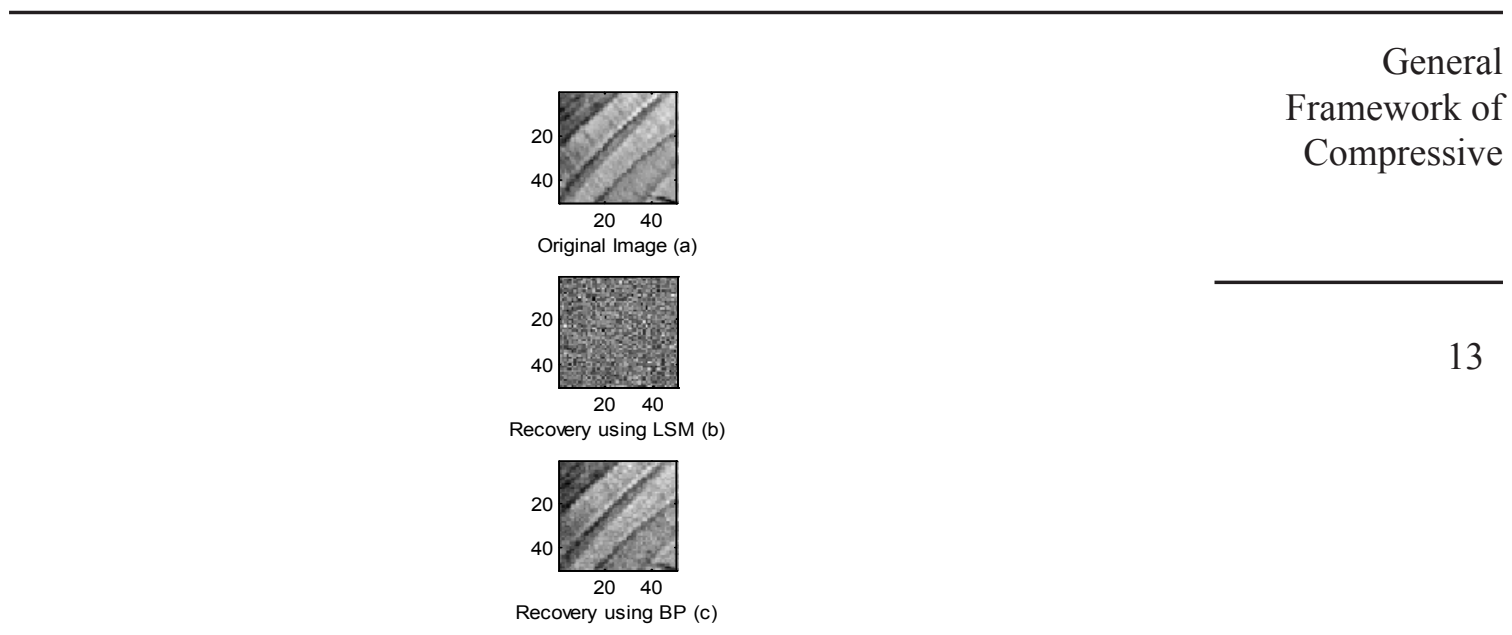

Figure 3: Original Image versus Recovered Image. (a) Original Signal. (b) Recovered image using least square Method. (c) Recovered image using Basic pursuit.

Fig.3. contains three images original image, recovered image using least square method and recovered image using basis pursuit. From here it is clear that image recovered using least square method is much distorted but image recovered using basic pursuit is almost similar to original image.

\section{CONCLUSION AND FUTURE SCOPE}

Compressive sampling appears to be a revolutionary technique for data acquisition and successful reconstruction. We implemented this technique for one dimensional signal as well as two dimensional image signal and successfully recover them from compressive random measurements. We analyzed recovery error due to variations in sparsity level and compression ratio and assured that successful reconstruction of signal relies on sparsity level and compression ratio. In future scope, we can use this technique for compression of other kind of one dimensional or multidimensional signal if they are sparse in some domain. This technique can play important role in microwave applications where sampling rate is very high due to high frequencies.

\section{REFERENCES}

Simon Haykin (2001) "Communication Systems", $4^{\text {th }}$ ed. NewYork:John Wiley and Sons.

D. Donoho (2006) “Compressed Sensing”, IEEE Trans. Inform. Theory, Vol. 52:4, pp. 12891306. http://dx.doi.org/10.1109/TIT.2006.871582.

E. Candès, J. Romberg, and T. Tao (2006) "Robust Uncertainty Principles: Exact Signal Reconstruction from Highly Incomplete Frequency Information," IEEE Trans. Inform. 
Thakur, P.
Theory, Vol. 52:2, pp. 489-509.

E.J.Candes (2006) "Compressive sampling,"in Proceedings of the International Congress of Mathematicians, Vol. 3, pp. 1433-1452.

J. Tropp and A.C. Gilbert (2007) "Signal Recovery from Partial Information Via Orthogonal Matching Pursuit," IEEE Trans. Inform. Theory, Vol. 53:12, pp. 4655- 4666.

Emmanuel Cand'es and Terence Tao (2005) "Decoding by Linear Programming. IEEE Trans. Inform. Theory, Vol 51:12, pp.4203-4215. http://dx.doi.org/10.1109/TIT.2005.858979.

Scott Shaobing Chen, David L. Donoho, and Michael A. Saunders (1999) "Atomic Decomposition By Basis Pursuit. SIAM J. Sci Comp., 20:1, pp. 33-61.

E. Candès and J. Romberg (2007) "Sparsity and Incoherence in Compressive Sampling," Inverse Prob., Vol. 23:3, pp. 969-985. 